\title{
Mycosphaerella graminicola LysM Effector-Mediated Stealth Pathogenesis Subverts Recognition Through Both CERK1 and CEBiP Homologues in Wheat
}

\author{
Wing-Sham Lee, Jason J. Rudd, Kim E. Hammond-Kosack, and Kostya Kanyuka \\ Wheat Pathogenomics Team, Plant Biology and Crop Science Department, Rothamsted Research, Harpenden, AL5 2JQ, U.K. \\ Submitted 16 July 2013. Accepted 6 September 2013.
}

Fungal cell-wall chitin is a well-recognized pathogen-associated molecular pattern. Recognition of chitin in plants by pattern recognition receptors activates pathogen-triggered immunity (PTI). In Arabidopsis, this process is mediated by a plasma membrane receptor kinase, CERK1, whereas in rice, a receptor-like protein, $\mathrm{CEBiP}$, in addition to CERK1 is required. Secreted chitin-binding lysin motif (LysM) containing fungal effector proteins, such as Ecp6 from the biotrophic fungus Cladosporium fulvum, have been reported to interfere with PTI. Here, we identified wheat homologues of $C E R K 1$ and $C E B i P$ and investigated their role in the interaction with the nonbiotrophic pathogen of wheat Mycosphaerella graminicola (synonym Zymoseptoria tritici). We show that silencing of either $C E R K 1$ or $C E B i P$ in wheat, using Barley stripe mosaic virus-mediated virus-induced gene silencing, is sufficient in allowing leaf colonization by the normally nonpathogenic $M$. graminicola Mg3LysM (homologue of Ecp6) deletion mutant, while the Mg1LysM deletion mutant was fully pathogenic toward both silenced and wild-type wheat leaves. These data indicate that Mg3LysM is important for fungal evasion of PTI in wheat leaf tissue and that both CERK1 and CEBiP are required for activation of chitin-induced defenses, a feature conserved between rice and wheat, and perhaps, also in other cereal species.

Plants have evolved the ability to detect potentially pathogenic microorganisms by recognizing conserved pathogenderived signals known as pathogen-associated molecular patterns (PAMPs), usually via pattern-recognition receptors (PRR) that are expressed on the surface of plant cells (Jones and Dangl 2006). Recognition of PAMPs by the PRR triggers downstream signaling, which results in the activation of plant defense responses, a process referred to as PAMP-triggered immunity (PTI). Pathogenic microbes, on the other hand, have evolved specialized secreted effector proteins that are able to interfere with or suppress the activation of PTI, thus enabling the pathogen to successfully evade this first layer of plant defense (Gimenez-Ibanez et al. 2009; Göhre et al. 2008; Zhang et al. 2010). These secreted effectors are typically relatively

Corresponding author: K. Kanyuka; Telephone: +44 1582 763133; E-mail: kostya.kanyuka@rothamsted.ac.uk

* The $\boldsymbol{e}$-Xtra logo stands for "electronic extra" and indicates that 11 supplementary figures and nine supplementary tables are published online.

(C) 2014 The American Phytopathological Society small proteins and have, in many cases, been shown to function as virulence factors (de Jonge et al. 2011). Plants have also evolved disease resistance proteins that recognize, either directly or indirectly, pathogen effectors or the activity of these effectors, which results in activation of a second layer of defense known as effector-triggered immunity (Boller and $\mathrm{He}$ 2009).

One of the fungal PAMPs recognized by both plants and animals is chitin, a major component of fungal cell walls. In Arabidopsis thaliana, chitin recognition and signaling is mediated by CERK1 (chitin elicitor receptor kinase 1). Arabidopsis CERK1 contains three lysin motifs (LysM) that mediate binding to chitin-derived oligosaccharides (Miya et al. 2007). In rice (Oryza sativa), a second LysM-containing protein called CEBiP (chitin elicitor binding protein) is required in addition to CERK1 for chitin elicitor perception and signaling (Fig. 1A) (Kaku et al. 2006; Shimizu et al. 2010). This is in contrast to Arabidopsis, in which CERK1 alone appears to be sufficient for chitin-induced activation of defenses (Shinya et al. 2012). However, it has recently been suggested that, in this plant species, a homologue of rice CEBiP called LYM2 mediates a decrease in molecular flux between cells in the presence of chitin via a pathway that is independent of PTI and does not require CERK1 (Faulkner et al. 2013). Another recent report suggests that Arabidopsis LYM2 is not involved in chitin signaling but contributes to CERK1-independent resistance against a necrotrophic fungus Alternaria brassicicola via a novel diseaseresistance mechanism (Narusaka et al. 2013). Several pathogenic fungi have been shown to secrete LysM-containing effector proteins that are able to interfere with chitin-induced plant defense responses, such as the Ecp6 protein from the biotrophic fungal pathogen of tomato Cladosporium fulvum, which binds chitin with ultrahigh affinity (de Jonge and Thomma 2009; de Jonge et al 2010; Mentlak et al. 2012; Sánchez-Vallet et al. 2013). Ecp6 homologue-expressing fungal species include the economically important fungal pathogen Mycosphaerella graminicola (anamorph Septoria tritici, recently renamed Zymoseptoria tritici [Quaedvlieg et al. 2011]), the causal agent of Septoria tritici blotch (STB) disease of wheat.

STB is recognized as one of the most economically important diseases of wheat in the U.K. and western Europe and a threat to wheat crop yields worldwide (Dean et al. 2012). $M$. graminicola infects only the leaves of wheat (Triticum spp.) plants, entering the leaf via open stomata, after which the fungal hyphae grow apoplastically between the mesophyll cells in an extended symptomless infection phase of between 7 to 28 days, depending on the particular wheat genotype-fungal isolate combination. This extended symptomless phase is followed 
by rapid induction of host cell death and a subsequent necrotrophic feeding phase that is required for fungal asexual sporulation (Dean et al. 2012; Kema et al. 1996). Notably, fungal biomass does not increase significantly until the latter necrotrophic phase (Keon et al. 2007). Previous studies indicate that two homologues of $C$. fulvum Ecp6 identified in the M. graminicola genome, Mg3LysM and Mg1LysM, are capable of binding chitin and are also able to protect fungal hyphae against plant-derived chitinases (Marshall et al. 2011). In $C$. fulvum, the latter function is fulfilled by a separate effector protein Avr4 rather than by Ecp6 (van den Burg et al. 2006). Analyses of $M$. graminicola single-gene deletion mutant strains demonstrated that only Mg3LysM but not Mg1LysM is important for fungal virulence, through a role in preventing activation of chitin-triggered plant defenses. This indicated that chitin-induced PTI is important in STB disease development (Marshall et al. 2011), although the components of chitin recognition and downstream signaling pathways in wheat have not yet been characterized. It is worth noting that, even for the well-studied tomato-C. fulvum pathosystem, the nature of the one or more tomato receptors that compete for chitin binding with fungal Ecp6 remains unknown (Sánchez-Vallet et al. 2013).

In this study, we demonstrated that Barley stripe mosaic virus-mediated virus-induced gene silencing (BSMV-VIGS) (Lee et al. 2012) is relatively long-lasting and, therefore, can be applied for functional analysis of wheat genes involved in the interaction with plant pathogens that have a long symptomless infection stage, such as $M$. graminicola. Using in silico analyses, we identified the most likely wheat (Triticum aes- tivum) orthologues of CERK1 and CEBiP and, then, explored whether these two genes play a role during $M$. graminicola infection of wheat. Our results indicate that Mg3LysM is important for fungal evasion of PTI activation in the host tissue and that both $C E R K 1$ and $C E B i P$ are required for chitin recognition and signaling in wheat.

\section{RESULTS}

\section{Identification of rice homologues of $C E R K 1$ and $C E B i P$ in wheat.}

We searched the National Center for Biotechnology Information (NCBI) and Dana-Farber Cancer Institute (DFCI) Wheat Gene Index databases to find potential homologues of the rice $C E R K 1$ and $C E B i P$ genes in wheat. One wheat candidate unigene was identified for CERK1 (Ta.25461) and one for CEBiP (Ta.58242). When this project was initiated in early 2012, comprehensive bioinformatics analyses using the publicly available sequence resources, i.e., NCBI genomic and expressed sequence tag (EST) databases, DFCI Wheat Gene Index database, and an International Wheat Genome Sequencing Consortium (IWGSC) chromosome arm survey sequence repository at URGI INRA (Unité de Recherche Génomique Info Institut National de la Recherche Agronomique) (Versailles, France) led to the identification of only one candidate gene for CERK1 (UniGene Ta.25461; GenBank entry AK331369; predicted to reside on the short arm of homoeologous group 7 chromosomes) and one for CEBiP (UniGene Ta.73435; predicted to reside on the long arm of homoeologous group 4 chromosomes). The sequences of wheat and rice CERK1 pro-
A

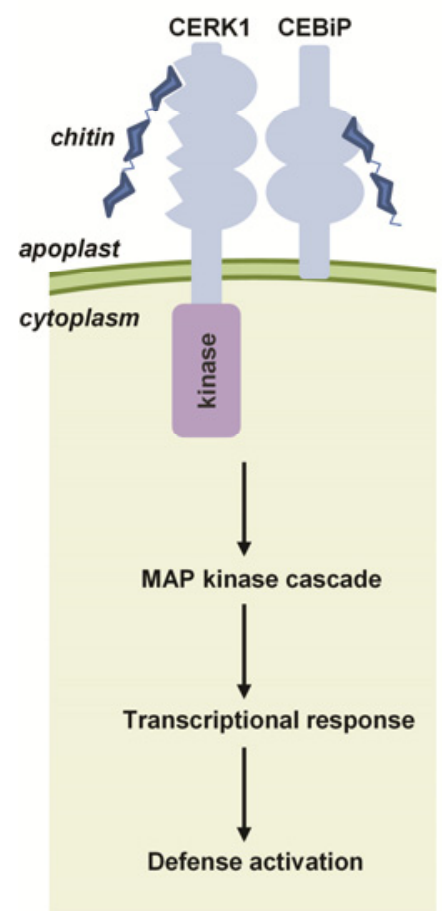

B

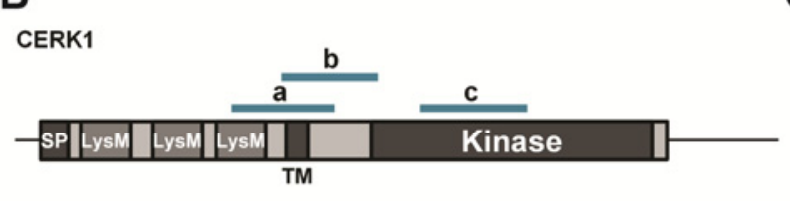

CEBiP
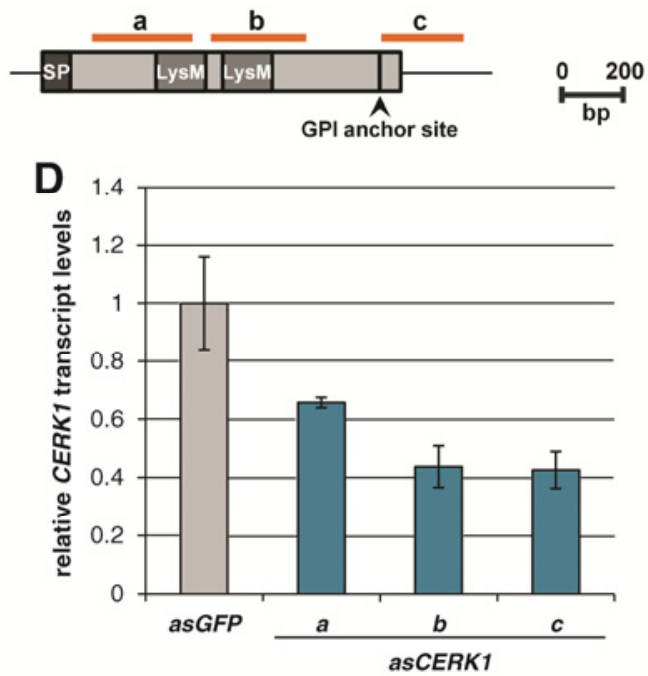

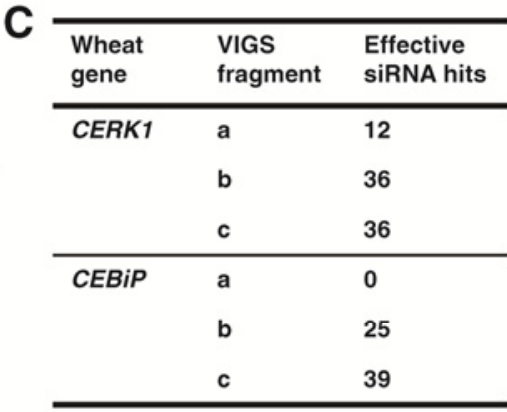

E

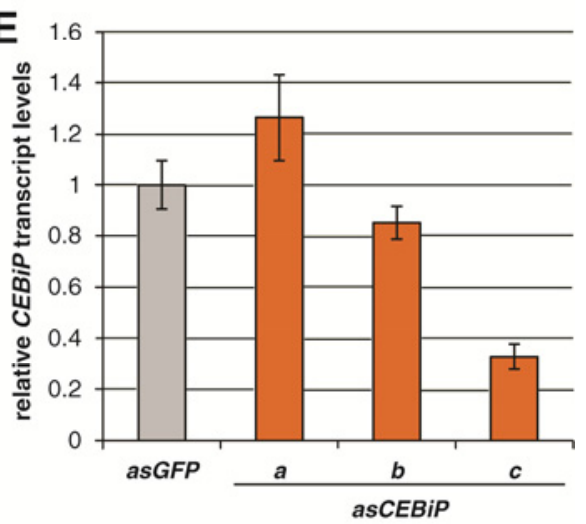

Fig. 1. Silencing predicted components of the chitin signaling pathway in wheat. A, Recognition of chitin fragments by rice CERK1 and CEBiP is thought to trigger downstream signaling, resulting in defense activation. B, Diagrammatical representation of wheat CERK1 and CEBiP. Bars extending from the termini of the predicted protein structures indicate nontranslated cDNA regions up- and downstream of the coding regions. Bars above each gene model indicate regions amplified to generate different Barley stripe mosaic virus-mediated virus-induced gene silencing (BSMV-VIGS) constructs targeting these genes. C, Numbers of effective siRNA hits predicted by Si-Fi software to arise from each of the gene fragments used in BSMV-VIGS. D and $\mathbf{E}$, Quantitative reverse transcription-polymerase chain reaction analysis of CERK1 and CEBiP transcript levels in BSMV-infected wheat plants at 14 days postinoculation. Data shown represents one of three independent experiments. Error bars in D and E represent mean \pm standard error of the means of three biological replicates. SP = signal peptide, $\mathrm{LysM}=$ lysin motif, TM = transmembrane region, GPI anchor site $=$ putative glycosylphosphatidylinositol modification site. 
teins share $86 \%$ amino acid identity and are predicted to contain the same structural features, namely an N-terminal signal peptide, three LysM motifs, a transmembrane domain, and a $\mathrm{C}$-terminal kinase domain. The sequences of wheat and rice CEBiP proteins are also very similar and share $81 \%$ amino acid identity. Both contain a signal peptide at the $\mathrm{N}$ terminus, two LysM motifs and a putative glycosylphosphatidylinositol (GPI) anchor site at the C-terminus (Supplementary Figs. S1 to $\mathrm{S} 3$ ).

Gene expression analysis revealed that both predicted chitin receptor genes $C E R K 1$ and $C E B i P$ were significantly up-regulated in susceptible wheat leaves at approximately 9 days postinoculation (dpi) with M. graminicola isolate IPO323, when the fungus begins to transition from biotrophic to necrotrophic growth (Supplementary Fig. S4). This is consistent with the function of these genes in activation of the PTI responses during the infection-phase transition (Rudd et al. 2008; unpublished).

\section{VIGS of $C E R K 1$ and $C E B i P$ in wheat.}

In order to study the role of CERKI and $C E B i P$ in the wheat-M. graminicola interaction, we used BSMV-VIGS to target each of these two genes for silencing. Three different fragments for each target gene were selected for VIGS construct preparation, aided by the siRNA-Finder (si-Fi) software (Fig. 1B and C). Off-target silencing was not predicted to arise from any of the gene fragments. Efficient knock-down of CERK1 transcript levels was achieved with all three of the VIGS constructs designed, with a reduction in target transcript levels of around $35 \%$ with construct 'a' to $56 \%$ with constructs ' $b$ ' and ' $c$ ' in the third leaves of wheat plants subjected to BSMV-VIGS when sampled at 14 dpi (Fig. 1D). The relative degree of silencing induced by each construct agreed well with the si-Fi software predictions (Fig. 1C). Similar data were obtained in three independent experiments, although the exact degree of silencing varied slightly between experiments, ranging from 20 to $40 \%$ reduction in CERK1 transcript levels in construct a-infected plants and from 45 to $70 \%$ reduction in construct b- or c-infected plants when sampled between 14 and 25 dpi (data not shown). Robust silencing of CERKI was still detectable at $28 \mathrm{dpi}$, with around $34 \%$ reduction in CERK1 transcript levels in construct a-infected plants and $46 \%$ reduction in construct b-infected plants (Supplementary Fig. S5).

Only two of the three BSMV-VIGS constructs designed to target $C E B i P$ were effective. Constructs $\mathrm{b}$ and $\mathrm{c}$ induced 15 and $67 \%$ reduction, respectively, of wheat $C E B i P$ transcript levels in the third leaves of infected plants sampled at $14 \mathrm{dpi}$ (Fig. 1E). This was, again, consistent with the si-Fi software predictions, as construct a was not predicted to generate any effective small interfering RNAs (Fig. 1C). Similar degrees of silencing with each $C E B i P$-specific VIGS construct were seen in three independent experiments in leaf samples taken between 14 and 24 dpi (data not shown).

In all subsequent experiments involving fungal inoculations, we used only the two VIGS constructs for each target gene, $C E R K 1$ and $C E B i P$, that provided the greatest degree of silencing.

\section{Optimization of BSMV-VIGS}

\section{for studying wheat leaf-M. graminicola interactions.}

We proceeded to explore the effect of transiently silencing $C E R K 1$ or $C E B i P$ on various types of wheat $-M$. graminicola interactions. Plants were preinoculated with the different BSMV-VIGS constructs at the two-leaf seedling stage, and between 10 to 14 days later, the third leaves inoculated with the wild-type strain M. graminicola IPO323 in an attached leaf bioassay (Rudd et al. 2008). Disease was assessed at 21 days post-fungal inoculation by scoring the area of $M$. graminicolainoculated leaf tissue that was both necrotic and evenly covered by fungal pycnidia as a percentage of the total M. graminicola-inoculated leaf area (hereafter referred to as percent pycnidial coverage). Leaves or areas of leaf tissue that displayed necrotic symptoms but developed no or only a few scattered pycnidia were scored as 'no pycnidia' areas.

Very few or no fungal pycnidia formed on either the $C E R K 1$ - or CEBiP-silenced or control BSMV:asGFP-inoculated wheat cv. Cadenza, which is resistant to M. graminicola IPO323, indicating that preinfection with BSMV did not compromise resistance to $M$. graminicola in these plants (Supplementary Fig. S6). There was also no significant difference in percent pycnidial coverage $\left(F_{15,70}=1.04, P=0.427\right)$ in leaves of wheat cv. Riband (susceptible to $M$. graminicola IPO323) preinoculated with the different BSMV-VIGS constructs (Fig. $2 \mathrm{~A}$ and B). Pycnidial coverage of the majority of cv. Riband leaves was more than $60 \%$ for all treatments and, therefore, indicated that the compatible wheat- $M$. graminicola interaction also remained unaltered. This experiment was repeated three more times with both cv. Cadenza and cv. Riband with consistent results.

\section{Silencing either $C E R K 1$ or $C E B i P$ allows wheat leaf colonization by the normally nonpathogenic M. graminicola $\triangle M$ M 3LysM mutant.}

We previously showed that the $M$. graminicola mutant strain $\triangle M g l$ Lys $M$ is fully virulent on leaves of wheat cv. Riband and also lacked the ability to block chitin-elicited defense responses (Marshall et al. 2011). When we inoculated CERK1- or CEBiPsilenced and control cv. Riband plants with the $\triangle M g l L y s M$ mutant strain, similar levels of disease were observed on silenced and nonsilenced leaves (Fig. 2C and D), with no significant difference in percent pycnidial coverage on plants preinoculated with the different BSMV-VIGS constructs $\left(F_{15,40}=\right.$ $0.91, P=0.564)$.

We then investigated whether silencing either CERK1 or $C E B i P$ would fully or partially restore the disease-causing ability of the $M$. graminicola mutant strain $\triangle M g 3 L y s M$. When the cv. Riband leaves were examined at 21 days post-fungal inoculation, significant differences were found between the percent pycnidial coverage profiles on leaves of plants that received treatments with the different BSMV-VIGS constructs $\left(F_{15,80}=3.67, P<0.001\right)$. As expected, few or no pycnidia were detected on leaves of most of the virus-free or BSMV:asGFP-infected control plants (Fig. 2E). By contrast, a significant proportion of the CERKI- or CEBiP-silenced leaves displayed high $(>60 \%) \triangle M g 3 L y s M$ pycnidial coverage $(P<0.05$, Fisher's least significant difference test) (Fig. $2 \mathrm{E}$ and $\mathrm{F})$.

\section{DISCUSSION}

We have previously shown that Mg3LysM is required for full virulence of the fungus $M$. graminicola (also known as Zymoseptoria tritici) on wheat leaves and that this effector protein is able to block the induction of chitin-elicited defenses in tomato cell cultures (Marshall et al. 2011). In this study, we demonstrate that Mg3LysM is required during the early asymptomatic phase of STB disease on wheat and has the role of shielding fungal chitin from recognition by the wheat chitin receptor proteins CERK1 and CEBiP. This is the first study to show that both CERK1 and CEBiP are involved in chitin perception in wheat and, also, that BSMV-VIGS can be used in wheat for identification of host defense components in the wheat $-M$. graminicola interaction. We show that infection of wheat leaves with the control viral vector does 
not, in itself, interfere with the outcome of either the incompatible (resistance) or compatible (disease) interaction with $M$. graminicola. These new data indicate that this transient gene-silencing approach is suitable for investigating and functionally characterizing the plant genes involved in both interaction outcomes.

In Arabidopsis, the receptor kinase CERK1 alone is sufficient for chitin perception, whereas in rice CERK1 and CEBiP cooperatively regulate chitin signaling (Shimizu et al. 2010; Shinya et al. 2012). It has previously been shown that Slp1, a homologue of $C$. fulvum Ecp6 from the rice blast fungus Magnaporthe oryzae, is able to directly compete with the rice CEBiP receptor for chitin binding (Mentlak et al. 2012) and that the Magnaporthe oryzae $\Delta$ slpl mutant was able to cause full rice blast disease in stable transgenic rice lines in which $C E B i P$ had been silenced by RNAi. It remains unknown whether CERK1 also plays a role in the Magnaporthe oryzaerice interaction. However, this is conceivable, because rice CEBiP and CERK1 have been shown to interact with each other through the extracellular LysM domains in the yeast two-hybrid system assays and are speculated to form functional heteroduplexes in planta (Fig. 1A) (Shimizu et al. 2010). Whether chitin signaling is coregulated by CERK1 and CEBiP in other monocotyledonous species has not yet been reported. It has, however, been shown that barley $C E B i P$ is involved in resistance to the mutant strain of rice blast pathogen Magnaporthe oryzae, called mossdl, that lacks the function of the SSD1 gene (a regulator of cell-wall assembly). The mossdl mutant was able to form appressoria and primary hyphae but, postinvasion, was severely restricted in pathogenicity towards leaf sheaths of compatible rice plants due to the activation of a plant hypersensitive cell-death response (Tanaka et al. 2007). However, when inoculated onto barley plants in which CEBiP had been silenced, either through stable RNAi or through BSMV-VIGS, this mutant was able to produce small disease lesions at a small proportion of inoculation sites, indicating that $C E B i P$ in barley contributes towards the host resistance to mossdl (Tanaka et al. 2010). Our data suggest that, in wheat as in rice, chitin recognition and signaling is coregulated by CERK1 and CEBiP and represents an evolutionarily conserved perception mechanism shared between rice and wheat and, also perhaps, in other cereal species.

In this study, we demonstrate that the $\triangle M g 3 L y s M$ mutant of $M$. graminicola, which is severely compromised in its disease-causing ability on wheat, is fully pathogenic on wheat leaves in which either $C E R K 1$ or $C E B i P$ have been silenced. On the other hand, silencing $C E R K 1$ or $C E B i P$ had no effect on the pathogenicity of the $\triangle 1 M g L y s M$ mutant toward wheat leaves. This is consistent with our previous observation that, although both $M$. graminicola LysM effectors Mg1LysM and Mg3LysM are able to bind chitin and protect fungal hyphae against hydrolytic plant enzymes, only Mg3LysM is able to block chitin-induced plant defense responses (Marshall et al. 2011). Our new data, therefore, suggests indirectly that the chitin-binding function of Mg3LysM during disease development is likely to be more important than its protective activity against plant chitinases.

M. graminicola has an infection biology that is different from that of another dothideomycete, C. fulvum, and to the more distantly related sordariomycete Magnaporthe oryzae. These are the only two fungal species to date that have been investigated with respect to LysM effectors and suppression or evasion of chitin-triggered plant immunity. While C. fulvum, like $M$. graminicola, invades host tissue via stomata and grows strictly intercellularly, it is a true biotrophic pathogen that is able to increase its biomass and sporulate in association with living plant cells (Thomma et al. 2005). Magnaporthe oryzae is a hemibiotroph that, in contrast to $M$. graminicola and $C$. fulvum, uses specialized infection structures called appressoria to penetrate inside plant cells. The Magnaporthe oryzae hyphae then undergo an extensive intracellular biotrophic phase during which the fungus is able to obtain nutrition from living plant cells before inducing extensive host cell death and lesion formation (Wilson and Talbot 2009).

Given the length of the early symptomless phase of $M$. graminicola infection, which persisted for 10 to 14 days in our experimental system, it is noteworthy that we have been able to use BSMV-VIGS to silence the wheat chitin receptor genes and study the in planta role of both in the infection process. Prior to this study, BSMV-VIGS has only been used to study plantpathogen interactions in which fungus-induced disease symptoms could be scored from 5 days (in the case of powdery mildew disease [Hein et al. 2005]) to 12 days (as with leaf or stem rust diseases [Scofield et al. 2005; Zhang et al. 2009]) postfungal inoculation. However, here, we were able to detect and utilize effective gene silencing through VIGS when scoring STB disease symptoms at 21 days post-fungal inoculation (corresponding to between 31 to 33 days post-virus inoculation). It was not possible to determine whether silencing of CERK1 and $C E B i P$ was retained in $M$. graminicola-infected wheat leaves during the latter stages of $M$. graminicola infection as, by this point, the host tissue is undergoing or has already undergone cell death. However, we were able to ascertain that silencing of CERK1 was still effective in the third leaves of virus-infected leaves sampled at 28 days post-virus inoculation, although we did not investigate whether silencing of $C E B i P$ persisted beyond 24 days post-virus inoculation.

This indicates that BSMV-VIGS may be suitable for functional gene studies involving other pathogens of monocotyledonous plant species with prolonged life cycles, for example, other members of the Mycosphaerella genus. Importantly, the efficiency of silencing induced by the different VIGS constructs targeting different parts of the same gene agreed well with that predicted by the si-Fi software. Even though only a small number of constructs were tested in this study, this good correspondence between experimental prediction and data generated suggests that si-Fi could be used to speed up the process of identifying effective target gene fragments for VIGS experiments and to minimize the likelihood of offtarget silencing.

One possible caveat in this study is that we analyzed only one homologue of rice CERK1 and only one homologue of rice $C E B i P$, predicted to locate on the short arm of homoeologous group 7 chromosomes and the long arm of homoeologous group 4 chromosomes in wheat, respectively. These genes were identified in our bioinformatics study conducted in early 2012. Since then, many new wheat sequences have been deposited into the NCBI EST database and IWGSC wheat chromosome arm survey sequence repository. Our recent bioinformatics analyses (August 2013) identified an additional homologue for each of the LysM-containing receptor genes in wheat. The CERK1-like gene is predicted to reside on the long arm of homoeologous group 6 chromosomes, whereas the CEBiP-like gene is predicted to reside on the long arm of homoeologous group 5 chromosomes. The two wheat $C E B i P$ homologues share 38 and $55 \%$ identity at the amino acid and nucleotide levels, respectively (Supplementary Fig. S7; data not shown). In silico analysis using si-Fi software predicts that none of the BSMV-VIGS constructs should be efficient in silencing the newly identified $C E B i P$ gene from the homoeologous chromosome group 5 (Supplementary Fig. S8). On the other hand, the two wheat CERK1 homologues are very similar and share 83 and $88 \%$ identity at the amino acid and nucleotide levels, respectively 
(Supplementary Fig. S9; data not shown). It is, therefore, likely that each of the three BSMV-VIGS constructs used in this study was efficient in silencing both wheat CERK1 homologues (Supplementary Fig. S10). Interestingly, each of the newly identified $C E R K 1$ and $C E B i P$ homologues is represented by only two to four partial EST, suggesting a very low level of expression. Therefore, our current work poses a number of questions that would be interesting to answer in follow-up studies. In particular, i) do the newly discovered CERK1 and CEBiP homologues represent functional genes or pseudogenes? ii) what is the function of the CEBiP homologue locating on the homoeologous chromosome group 5 and does this sequence play a role in the $M$. graminicola-wheat interaction? and iii) are the two CERKI wheat homologues functionally redundant or is only one of them important during $M$. graminicola infection on wheat?

In summary, our data indicate that CERK1- and CEBiPcoregulation of chitin elicitor detection has been conserved within cereal species and that evasion of this plant immune response has been conserved between fungi with very different in planta lifestyles. Putative LysM-containing effector proteins have been identified in both pathogenic and saprophytic fungal species across five different phyla (de Jonge and Thomma 2009), and it is possible that, at least in many of these pathogenic species, LysM-containing effector proteins are similarly involved in subversion of host recognition of essential fungal cell-wall components.

\section{MATERIALS AND METHODS}

Sequence analysis, alignment, and domain predictions.

To identify CERK1 and CEBiP homologues in wheat, the NCBI, DFCI Wheat Gene Index, and an International Wheat Genome Sequencing Consortium chromosome survey sequence repository at URGI INRA databases were used. The nucleotide and amino acid sequences of rice CERK1 and CEBiP proteins were used to search for their homologues in wheat using the BLASTN and TBLASTN programs. At the start of this project in early 2012, it was possible to identify only one candidate full-length coding DNA sequence with high homology to the corresponding rice genes, and these were named wheat $C E R K 1$ and wheat $C E B i P$. Searches for wheat sequences with LysM motifs did not identify any other candidate LysM-containing sequences in the available databases. ClustalW was used for multiple sequence alignment of all known rice LysM receptor-like kinases and wheat CERK1 and for alignment of the rice and corresponding wheat candidate CEBiP amino acid sequences. The multiple sequence alignment was used to construct an unrooted maximum likelihood phylogenetic tree in
A

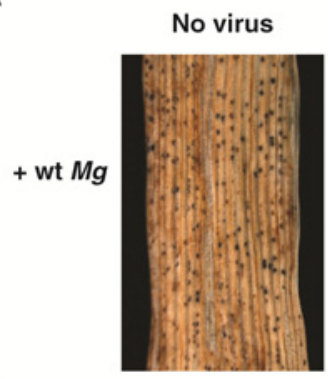

C

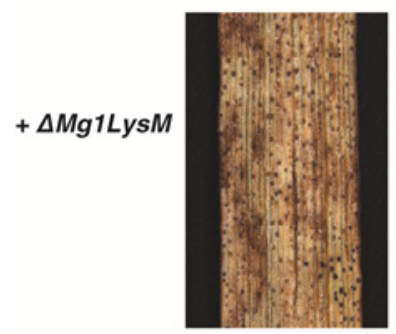

E

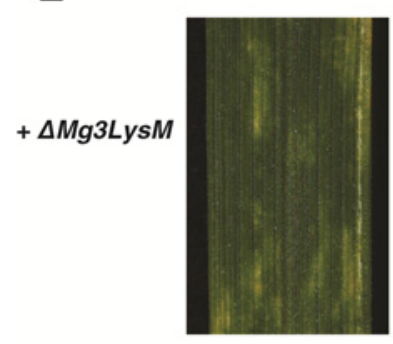

BSMV:asGFP
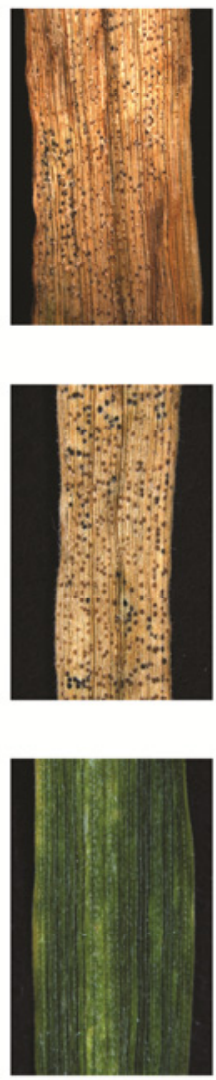
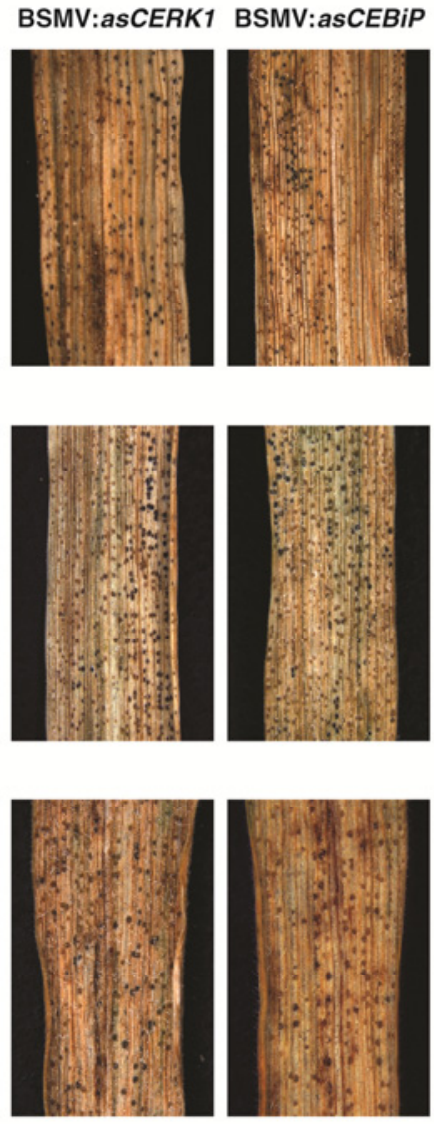

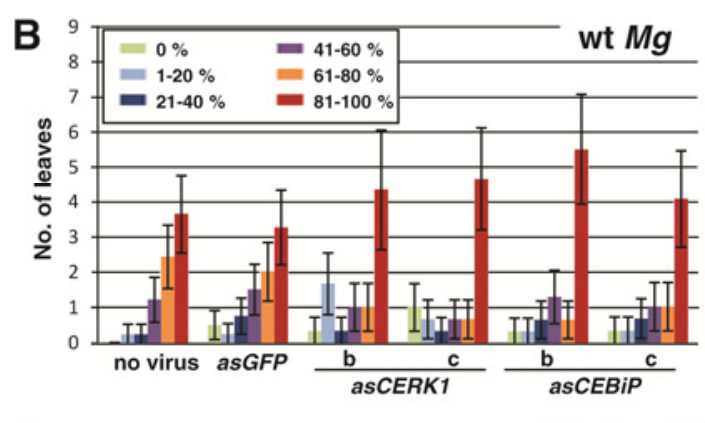

D
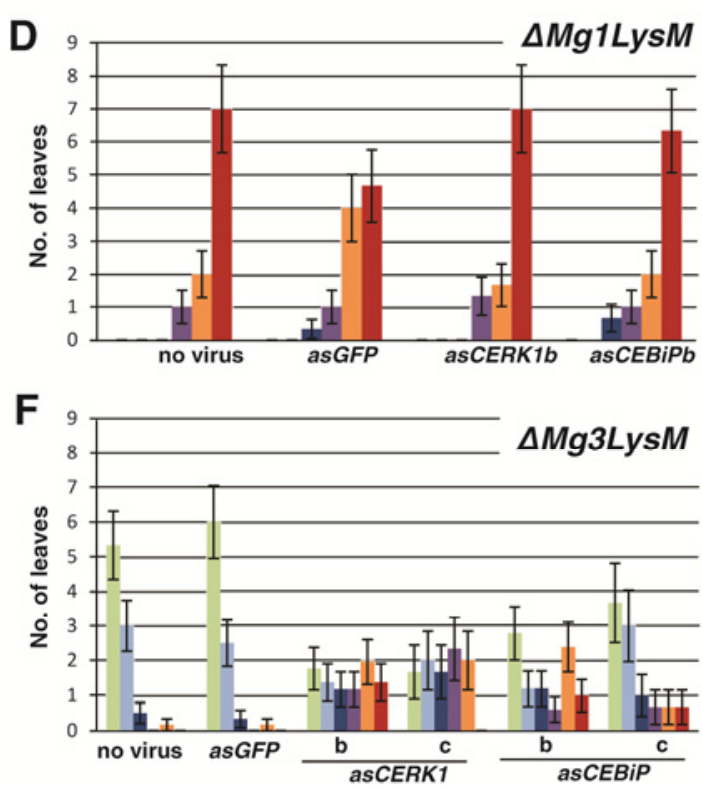

Fig. 2. The effect of silencing CERK1 and CEBiP on disease caused by wild-type Mycosphaerella graminicola IPO323 and the $\triangle M g 1 L y s M$ and $\triangle M g 3 L y s M$ mutants in wheat. The control virus treatments (no virus and BSMV:asGFP) and silencing constructs BSMV:asCERK1b, BSMV:asCERK1c, BSMV: asCEBiPb, and BSMV:asCEBiPc were used for these experiments. Disease symptoms and percent pycnidial coverage on $\mathbf{A}$ and $\mathbf{B}$, wild-type $M$. graminicola, $\mathbf{C}$ and $\mathbf{D}, \triangle M g l L y s M$ - and $\mathbf{E}$ and $\mathbf{F}, \triangle M g 3 L y s M$-inoculated leaves of wheat cv. Riband. Representative photographs of leaves and percent pycnidial coverage scores were taken at 21 days postinoculation. The percent pycnidial coverage was assessed as the area of $M$. graminicola-inoculated leaf tissue showing both necrosis and even coverage by fungal pycnidia as a percentage of the total inoculated leaf area. Ten leaves per M. graminicola strain and Barley stripe mosaic virus-mediated virus-induced gene silencing construct combination were scored in each experiment. The data plotted are the mean numbers of leaves within each of the six pycnidial coverage score classes, calculated using data pooled from three independent experiments. Error bars represent mean \pm standard error. 
MEGA5 (Tamura et al. 2011). Protein domain predictions were made using InterProScan and using the PROSITE database resource. All predictions were inspected and adjusted manually. GPI modification sites were predicted using the big-PI Plant Predictor (Eisenhaber et al. 2003).

\section{Plant growth conditions.}

Nicotiana benthamiana plants for preparation of BSMV sap inoculum and seedlings of wheat (Triticum aestivum) cultivars Riband and Cadenza were grown in a controlled environment chamber at $23^{\circ} \mathrm{C}, 60 \%$ relative humidity, with a $16-\mathrm{h}$ photoperiod (approximately $120 \mu \mathrm{mol} \mathrm{m}{ }^{-2}$ per second of light).

\section{Preparation of BSMV VIGS constructs and viral inoculations.}

The BSMV-VIGS system described by Yuan and associates (2011), comprising three T-DNA binary plasmids, pCaBS- $\alpha$, pCaBS- $\beta$, and pCa-ybLIC, was used. Gene-silencing constructs were created by cloning fragments of wheat $C E R K 1$ and $C E B i P$ fragments into $\mathrm{pCa}-\mathrm{\gamma bLIC}$ in antisense orientation, using a ligation-independent cloning strategy. In silico predictions by si-Fi software were used to select the most effective gene-specific fragments for silencing, ranging from 254 to 325 bp in size, and also to ensure the selected fragments were not likely to direct off-target silencing. The cDNA fragments were generated by standard reverse transcription-polymerase chain reaction (RT-PCR) from total RNA extracted from wheat cv. Cadenza leaf tissue using primers described in Supplementary Table S1. A 236-bp fragment of the green fluorescent protein (GFP) gene was amplified by PCR from the GFP-expression cassette plasmid pBIN35S::GFP4 (Haseloff et al. 1997), using the primer pair LIC asGFP F and LIC asGFP R, inserted into $\mathrm{pCa}-\mathrm{\gamma bLIC}$ in antisense orientation to generate the negative control construct for VIGS.

The BSMV pCaBS- $\alpha$, pCaBS- $\beta$, and pCa- $\mathrm{\gamma bLICv}$ derivatives were transformed separately into Agrobacterium tumefaciens GV3101 by electroporation. For agroinfiltration, single colonies were grown for 20 to $22 \mathrm{~h}$ at $28^{\circ} \mathrm{C}$ with constant shaking in $5 \mathrm{ml}$ of Luria Bertani containing $50 \mu \mathrm{g}$ of kanamycin per milliliter. Bacterial cells were pelleted by centrifugation at $2,400 \times g$ for $15 \mathrm{~min}$ at $4^{\circ} \mathrm{C}$ and were resuspended in agroinfiltration buffer $\left[10 \mathrm{mM} \mathrm{MgCl}_{2}, 10 \mathrm{mM}\right.$ 2-( $N$-morpholino) ethanesulfonic acid (MES), $\mathrm{pH} 5.6$, and $0.1 \mathrm{mM}$ acetosyringone] to a final optical density at $600 \mathrm{~nm}$ of 1.5 . After a 3-h incubation at room temperature, agrobacteria containing pCaBS- $\alpha$, pCaBS- $\beta$, and the relevant pCa- $\gamma$ bLIC derivative were mixed in a 1:1:1 ratio and were infiltrated into the abaxial side of the leaves of 3- to 4-week-old $N$. benthamiana plants with a $1-\mathrm{ml}$ needleless syringe. The infiltrated leaves were harvested at 3 to 4 days postinfiltration, were ground using a mortar and pestle in $10 \mathrm{mM}$ potassium phosphate buffer ( $\mathrm{pH}$ 6.8) containing 1\% celite, and the sap was used to mechanically inoculate the first leaf of 11-day-old wheat plants.

\section{Fungal strains and inoculations.}

The $M$. graminicola wild-type isolate IPO323 and its mutant derivatives $\triangle M g 3$ Lys $M$ and $\Delta M g l$ Lys $M$ (Marshall et al. 2011) were used in this study. Attached wheat leaf infection assays were done as described previously (Rudd et al. 2008), with slight modifications. The third leaves of 23-day-old wheat plants (corresponding to 12 days post-viral inoculation in which BSMV-infected plants were used) were fastened, adaxial side up, to a metal platform using double-sided sticky tape. The leaves were then inoculated evenly with fungal spores at a density of $5 \times 10^{6}$ cells per milliliter of water containing $0.1 \%$ (vol/vol) Silwet L-77 (Lehle Seeds, Round Rock, TX, U.S.A.) using cotton swabs. The plants were placed inside transparent boxes to retain high humidity for the first $72 \mathrm{~h}$ and were then returned to $60 \%$ relative humidity for up to 20 days. Disease was assessed at $21 \mathrm{dpi}$ by scoring the area of $M$. graminicolainoculated leaf tissue that was both necrotic and evenly covered by fungal pycnidia. The scores were sorted into six pycnidial coverage classes: $0 \%, 1$ to $20 \%, 21$ to $40 \%, 41$ to $60 \%$, 61 to $80 \%$, and 80 to $100 \%$. Data shown are the mean number of leaves within each pycnidial coverage class, calculated using combined data from three independent experiments. All disease assessments were made by the same person.

\section{RNA extraction and quantitative RT-PCR.}

When quantitative RT-PCR on $M$. graminicola-infected tissue was carried out, tissues were excised at various time points after inoculation, with five leaves pooled for each biological replicate, with each leaf harvested from a separate plant. All leaf bioassays were performed in triplicate.

Total RNA was extracted from freeze-dried leaves using TRI reagent (Sigma Aldrich, St. Louis) following the manufacturer's protocol. One microgram of total RNA was treated with RQ1 RNase-free DNase I (Promega, Madison, WI, U.S.A.) and was used for cDNA synthesis using High Capacity cDNA reverse transcription kit (Applied Biosystems, Foster City, CA, U.S.A.) according to the manufacturer's instructions. The resulting cDNA was diluted 1:20 with sterile deionized water and was analyzed using SYBR Green Jumpstart ReadyMix (Sigma Aldrich). A 5- $\mu$ l aliquot of diluted cDNA was used in a $20-\mu 1$ PCR reaction, with an annealing temperature of $60^{\circ} \mathrm{C}$. Quantification of gene expression was carried out in an ABI 7500 Real-Time PCR system (Applied Biosystems). For normalization of test gene expression using quantitative RT-PCR in silenced- versus virus control-infected leaves, three housekeeping genes, beta-tubulin, eIF4E and $C D C 48$, were initially considered. Beta-tubulin 1 and eIF4E are commonly used as reference genes in other organisms, and $C D C 48$ has been shown to be stably expressed in wheat under a range of environmental conditions (Paolacci et al. 2009). Pair-wise comparisons of the expression profiles of these three housekeeping genes in the third leaves of mockand virus-inoculated plants revealed $C D C 48$ as the most suitable reference for subsequent quantitative RT-PCR analyses (Supplementary Fig. S11). Moreover, CDC48 was stably expressed over the $M$. graminicola-wheat infection time course, indicating that this housekeeping gene could also be used as a reference gene for quantitative RT-PCR when studying wheat gene expression during $M$. graminicola infection (our unpublished RNA-Seq data). The primers and final primer concentrations used for real-time PCR are described in Supplementary Table S2.

\section{Statistical analyses.}

GenStat (release 14.1, 2011; VSN International Ltd, Hemel Hempstead, U.K.) was used for the statistical analyses. Differences in normalized CERK1 and CEBiP transcript levels between mock- and M. graminicola IPO323-inoculated wheat leaves were analyzed using REML linear mixed modeling. Significance of difference between mean transcript levels was determined using least significant differences (LSD) at the 5\% $(P<0.05)$ level of significance. Means and LSD values are displayed in Supplementary Tables S3 to S6. The percent pycnidial-coverage data were analyzed using generalized linear modeling assuming a Poisson distribution with a logarithm link function. The variate modeled was the number of leaves within each pycnidial coverage class for each BSMV-VIGS construct treatment group, accounting for the three experiments and seedling trays nested within experiments as blocking terms 
in the model. Significance of model terms was assessed using change in deviance, invoking approximate F-tests. Calculated mean values and standard errors were output and are displayed in Supplementary Tables S7 to S9. Significance of difference between calculated means was determined using LSD at the $5 \%$ level of significance.

\section{ACKNOWLEDGMENTS}

We would like to thank D. Li (State Key Laboratory of Agro-Biotechnology, Beijing, China) and A. Jackson (University of California, Berkeley, CA, U.S.A.) for providing binary BSMV clones and advice on the BSMV-VIGS system for cereals. We thank S. Powers (Rothamsted Research) for help with statistical analyses. The work was supported by the Biotechnology and Biological Sciences Research Council of the UK (BBSRC) through the Institute Strategic Program 20:20 Wheat. This research was carried out under the Fera agency of the U.K. Department for Environment, Food and Rural Affairs plant health license PHSI $181 / 6786$.

\section{LITERATURE CITED}

Boller, T., and He, S. Y. 2009. Innate immunity in plants: an arms race between pattern recognition receptors in plants and effectors in microbial pathogens. Science 324:742-744.

Dean, R., Van Kan, J. A., Pretorius, Z. A., Hammond-Kosack, K. E., Di Pietro, A., Spanu, P. D., Rudd, J. J., Dickman, M., Kahmann, R., Ellis, J., and Foster, G. D. 2012. The top 10 fungal pathogens in molecular plant pathology. Mol. Plant Pathol. 12:414-430.

de Jonge, R., and Thomma, B. P. 2009. Fungal LysM effectors: Extinguishers of host immunity? Trends Microbiol. 17:151-157.

de Jonge, R., van Esse, H. P., Kombrink, A., Shinya, T., Desaki, Y., Bours, R., van der Krol, S., Shibuya, N., Joosten, M. H. A. J., and Thomma, B. P. 2010. Conserved fungal LysM effector Ecp6 prevents chitin-triggered immunity in plants. Science 329:953-955.

de Jonge, R., Bolton, M. D., and Thomma, B. P. 2011. How filamentous pathogens co-opt plants: The ins and outs of fungal effectors. Curr. Opin. Plant Biol. 14:400-406.

Eisenhaber, B., Wildpaner, M., Schultz, C. J., Borner, G. H. H., Dupree, P., and Eisenhaber, F. 2003. Glycosylphosphatidylinositol lipid anchoring of plant proteins. Sensitive prediction from sequence- and genome-wide studies for Arabidopsis and rice. Plant Physiol. 133:16911701.

Faulkner, C., Petutschnig, E., Benitez-Alfonso, Y., Beck, M., Robatzek, S., Lipka, V., and Maule, A. J. 2013. LYM2-dependent chitin perception limits molecular flux via plasmodesmata. Proc. Natl. Acad. Sci. U.S.A. 110:9166-9170.

Gimenez-Ibanez, S., Hann, D. R., Ntoukakis, V., Petutschnig, E., Lipka, V., and Rathjen, J. P. 2009. AvrPtoB targets the LysM receptor kinase CERK1 to promote bacterial virulence on plants. Curr. Biol. 19:423-429.

Göhre, V., Spallek, T., Heidrun, H., Mersmann, S., Mentzel, T., Boller, T., de Torres, M., Mansfield, J. W., and Robatzek, S. 2008. Plant patternrecognition receptor FLS2 is directed for degradation by the bacterial ubiquitin ligase AvrPtoB. Curr. Biol. 18:1824-1832.

Haseloff, J., Siemering, K. R., Prasher, D. C., and Hodge, S. 1997. Removal of a cryptic intron and subcellular localization of green fluorescent protein are required to mark transgenic Arabidopsis plants brightly. Proc. Natl. Acad. Sci. U.S.A. 94:2122-2127.

Hein, I., Barciszewska-Pacak, M., Hrubikova, K., Williamson, S., Dinesen, M., Soenderby, I. E., Sundar, S., Jarmolowski, A., Shirasu, K., and Lacomme, C. 2005. Virus-induced gene silencing-based functional characterization of genes associated with powdery mildew resistance in barley. Plant Physiol. 138:2155-2164.

Jones, J. D. G., and Dangl, J. L. 2006. The plant immune system. Nature 444:323-329.

Kaku, H., Nishizawa, Y., Ishii-Minami, N., Akimoto-Tomiyama, C., Dohmae, N., Takio, K., Minami, E., and Shibuya, N. 2006. Plant cells recognize chitin fragments for defense signalling through a plasma membrane receptor. Proc. Natl. Acad. Sci. U.S.A. 103:11086-11091.

Kema, G. H. J., Yu, D. Z., Rijkenberg, F. H. J., Shaw, M. W., and Baayen, R. P. 1996. Histology of the pathogenesis of Mycosphaerella graminicola in wheat. Phytopathology 86:777-786.

Keon, J., Antoniw, J., Carzaniga, R., Deller, S., Ward, J. L., Baker, J. M. Beale, M. H., Hammond-Kosack, K. E., and Rudd, J. J. 2007. Transcriptional adaptation of Mycosphaerella graminicola to programmed cell death of its susceptible wheat host. Mol. Plant-Microbe Interact. 20:178193.
Lee, W. S., Hammond-Kosack, K. E., and Kanyuka, K. 2012. Barley stripe mosaic virus-mediated tools for investigating gene function in cereal plants and their pathogens: Virus-induced gene silencing, host-mediated gene silencing, and virus-mediated overexpression of heterologous protein. Plant Physiol. 160:582-590.

Marshall, R., Kombrink, A., Motteram, J., Loza-Reyes, E., Lucas, J., Hammond-Kosack, K. E., Thomma, B. P. H. J., and Rudd, J. J. 2011. Analysis of two in planta expressed LysM effector homologs from the fungus Mycosphaerella graminicola reveals novel functional properties and varying contributions to virulence on wheat. Plant Physiol. $156: 756-769$

Mentlak, T. A., Kombrink, A., Shinya, T., Ryder, L. S., Otomo, I., Saitoh, H., Terauchi, R., Nishizawa, Y., Shibuya, N., Thomma, B. P. H. J., and Talbot, N. J. 2012. Effector-mediated suppression of chitin-triggered immunity by Magnaporthe oryzae is necessary for rice blast disease. Plant Cell 24:322-335.

Miya, A., Albert, P., Shinya, T., Desaki, Y., Ichimura, K., Shirasu, K., Narusaka, Y., Kawakami, N., Kaku, H., and Shibuya, N. 2007. CERK1, a LysM receptor kinase, is essential for chitin elicitor signalling in Arabidopsis. Proc. Natl. Acad. Sci. U.S.A. 104:19613-19618.

Narusaka, Y., Shinya, T., Narusaka, M., Motoyama, N., Shimada, H., Murakami, K., Shibuya, N. 2013. Presence of LYM2 dependent but CERK1 independent disease resistance in Arabidopsis. Plant Signal. Behav. 8:e25345. Published online.

Paolacci, A. R., Tanzarella, O. A., Porceddu, E., and Ciaffi, M. 2009. Identification and validation of reference genes for quantitative RT-PCR normalization in wheat. BMC Mol. Biol. 10:11.

Quaedvlieg, W., Kema, G. H., Groenewald, J. Z., Verkley, G. J., Seifbarghi, S., Razavi, M., Mirzadi Gohari, A., Mehrabi, R., and Crous, P. W. 2011 Zymoseptoria gen. nov.: A new genus to accommodate Septoria-like species occurring on graminicolous hosts. Persoonia 26:57-69.

Rudd, J. J., Keon, J., and Hammond-Kosack, K. E. 2008. The wheat mitogen-activated protein kinases TaMPK3 and TaMPK6 are differentially regulated at multiple levels during compatible disease interactions with Mycosphaerella graminicola. Plant Physiol. 147:802-815.

Sánchez-Vallet, A., Saleem-Batcha, R., Kombrink, A., Hansen, G., Valkenburg, D.-J., Thomma, B. P. H. J., and Mesters, J. R. 2013.Fungal effector Ecp6 outcompetes host immune receptor for chitin binding through intrachain LysM dimerization. eLife 2:e00790. Published online.

Scofield, S. R., Huang, L., Brandt, A. S., and Gill, B. S. 2005. Development of a virus-induced gene silencing system for hexaploid wheat and its use in functional analysis of the Lr21-mediated leaf rust resistance pathway. Plant Physiol. 138:2165-2173.

Scofield, S., and Nelson, R. 2009. Resources for virus-induced gene silencing in the grasses. Plant Physiol. 149:152-157.

Shimizu, T., Nakano, T., Takamizawa, D., Desaki, Y., Ishii-Minami, N., Nishizawa, Y., Minami, E., Okada, K., Yamane, H., Kaku, H., and Shibuya, N. 2010. Two LysM receptor molecules, CEBiP and OsCERK1, cooperatively regulate chitin elicitor signalling in rice. Plant J. 64:204214.

Shinya, T., Noriko, M., Asahi, I., Miyuki, W., Kota, K., Masahiro, H., Kaku, H., and Shibuya, N. 2012. Functional characterization of CEBiP and CERK1 homologs in Arabidopsis and rice reveals the presence of different chitin receptor systems in plants. Plant Cell Physiol. 53:696706.

Tamura, K., Peterson, D., Peterson, N., Stecher, G., Masatoshi, N., and Kumar, S. 2011. MEGA5: Molecular evolutionary genetics analysis using maximum likelihood, evolutionary distance and maximum parsimony methods. Mol. Biol. Evol. 28:2731-2739.

Tanaka, S., Yamada, K., Yabumoto, K., Fujii, S., Huser, A., Tsuji, G., Koga, H., Dohi, K., Mori, M., Shiraishi, T., O'Connell, R., and Kubo, Y 2007. Saccharomyces cerevisiae SSD1 orthologues are essential for host infection by the ascomycete plant pathogens Colletotrichum lagenarium and Magnaporthe grisea. Mol. Microbiol. 64:1332-1349.

Tanaka, S., Ichikawa, A., Yamada, K., Tsuji, G., Nishiuchi, T., Mori, M., Koga, H., Nishizawa, Y., O'Connell, R., and Kubo, Y. 2010. $H \nu C E B i P$, a gene homologous to rice chitin receptor $C E B i P$, contributes to basal resistance of barley to Magnaporthe oryzae. BMC Plant Biol. 10:288

Thomma, B. P. H. J., van Esse, H. P., Crous, P. W., and de Wit, P. J. G. M. 2005. Cladosporium fulvum (syn. Passalora fulva), a highly specialized plant pathogen as a model for functional studies on plant pathogenic Mycosphaerellaceae. Mol. Plant Pathol. 6:379-393.

van den Burg, H. A., Harrison, S. J., Joosten, M. H. A. J., Vervoort, J., and de Wit, P. J. G. M. 2006. Cladosporium fulvum Avr4 protects fungal cell wall against hydrolysis by plant chitinases accumulating during infection. Mol. Plant Microbe Interact. 19:1420-1430.

Wilson, R. A., and Talbot, N. J. 2009. Under pressure: Investigating the biology of plant infection by Magnaporthe oryzae. Nat. Rev. Microbiol. 
7:185-195.

Yuan, C., Li, C., Yan, L., Jackson, A. O., Liu, Z., Han, C., Yu, J., and Li, D. 2011. A high throughput Barley stripe mosaic virus vector for virusinduced gene silencing in monocots and dicots. PLoS ONE 6:e26468. Published online.

Zhang, J., Li, W., Xiang, T., Liu, Z., Laluk, K., Ding, X., Zou, Y., Gao, M., Zhang, X., Chen, S., Mengiste, T., Zhang, Y. and Zhou, J.-M. 2010. Receptor-like cytoplasmic kinases integrate signalling from multiple plant immune receptors and are targeted by a Pseudomonas syringae effector. Cell Host Microbe 7:290-301.

Zhang, L., Lavery, L., Gill, U., Gill, K., Steffenson, B., Yan, G., Chen, X., and Kleinhofs, A. 2009. A cation/proton-exchanging protein is a candidate for the barley NecS1 gene controlling necrosis and enhanced defense response to stem rust. Theor. Appl. Genet. 118:385-397.
AUTHOR-RECOMMENDED INTERNET RESOURCES

The CerealsDB (cereals database): www.cerealsdb.uk.net/CerealsDB/Documents/DOC_CerealsDB.php DFCI Wheat Gene Index database: compbio.dfci.harvard.edu/cgibin/tgi/gimain.pl?gudb=wheat

GPI Modification Site Prediction in Plants (big-PI Plant Predictor database): mendel.imp.ac.at/gpi/plant_server.html

National Center for Biotechnology Information Basic Local Alignment Search Tool: blast.ncbi.nlm.nih.gov/Blast.cgi

PROSITE (database of protein domains, families and functional sites): prosite.expasy.org

siRNA-Finder (si-Fi) software: labtools.ipk-gatersleben.de/index.html The Wheat Portal: wheat-urgi.versailles.inra.fr 